Article

\title{
The Vibrio cholerae Cytotoxin (VCC) Simultaneously Activates Responses of Death, Inflammation (NF-kB) and Survival (AP-1) through the MAPK Signaling in Human Macrophages THP-1
}

Julio R. Escartín-Gutiérrez ${ }^{1,2}$, Aldo Arturo Reséndiz Albor ${ }^{2}$, Carlos Alberto Castañón-Sánchez ${ }^{3}$, Rafael Campos-Rodríguez ${ }^{1 \dagger}$, Gustavo Pedraza-Alva ${ }^{4}$, Miguel Ángel Torres-Vega ${ }^{5}$ Paula Figueroa-Arredondo ${ }^{1}$

\footnotetext{
${ }^{1}$ Departamento de Investigación. Doctorado en Investigación en Medicina, Laboratorio de Investigación Dr. Rafael Campos Rodríguez, Escuela Superior de Medicina. Sección de Estudios de Posgrado e Investigación. Instituto Politécnico Nacional, México City 07320 /México.

${ }^{2}$ Laboratorio de Inmunidad de mucosas. Escuela Superior de Medicina. Sección de Estudios de Posgrado e Investigación. Instituto Politécnico Nacional, México City 07320 /México.

${ }^{3}$ Laboratorio de Investigación Biomédica. Hospital Regional de Alta Especialidad de Oaxaca. Oaxaca Mexico,

${ }_{4}^{4}$ Departamento de Medicina Molecular y Bioprocesos, Instituto de Biotecnología, Universidad Nacional Autónoma de México, Cuernavaca, Morelos, México.

${ }^{5}$ Departamento de Gastroenterología, Instituto Nacional de Ciencias Medicas y Nutrición Salvador Zubirán.

${ }^{+}$Deceased
}

Correspondence should be addressed to Figueroa-Arredondo Paula.

paula figueroa@icloud.com, pfigueroaa@ipn.mx

\begin{abstract}
The human innate immune response to the pore-forming toxin of Vibrio cholerae VCC, is currently under study. Here, in vitro studies on a human macrophage cell line (THP-1), helped explore the activated pathways involved on the onset the innate immune response towards the cytotoxin. The secreted monomeric $65 \mathrm{KDa}$ form interacts with mature macrophages in $\mathrm{pg} / \mathrm{ml}$ concentrations, determined by dose response experiments after treatments under $1 \mathrm{~h}$. Non vacuolating concentrations $(\mathrm{pg} / \mathrm{ml})$ were applied to the cells; immunoblots revealed activation of MAPKs: early overexpression of p38 and ERK. Cell lysis by release of lactate dehydrogenase (LDH) was not apparent in the first hour, nonetheless it was positive after $24 \mathrm{~h}$. Finally, to discern whether the VCC stimulates transcriptional activators via MAPKs pathway, NF- $\kappa$ B and AP-1 were studied by real time quantitation. Increased expression of p50 (NF-kB), cJun and cFos (AP-1) was observed. Given that NF- $\kappa B$ is the transcription factor initiating inflammation of innate immune response and in turn, AP-1 is responsible for cell surviving response, results from this study lead us to conclude that VCC in vitro treatments, induce a pro-inflammatory and a surviving response, in less than one hour on activated macrophages.
\end{abstract}

Keywords: Vibrio cholerae cytotoxin, VCC, MAPKs, p38, ERK, pro-inflammatory, innate immune response, survival response.

Key Contribution: The monomeric form of VCC activates the MAPKs pathway in the first hour of interaction, leading the macrophage to the onset of a pro-inflammatory and pro-survival innate immune response. Lowering the concentration from ng to pg of toxin, it is possible to avoid the vacuolization process caused by the heptameric form of the toxin, and further observe the innate response of the monomeric cytotoxin. 


\section{Introduction}

The innate immune response is the first line of protection against pathogens and its components, get sensed by pattern recognition receptors (PRR). In turn, these receptors recognize pathogen-associated molecular patterns (PAMPs) for a prompt inflammatory response to begin $[1,2]$. Eukaryotic cells rely on the family of toll-like receptors (TLRs), which are the innate immune receptors, best characterized in macrophages, since macrophages perform in the first line of immune response (altogether with dendritic cells). It is known that TLRs stimulation activates signalling pathways such as mitogen activated protein kinases (MAPK) and the nuclear transcription factor $\mathrm{NF}-\mathrm{kB}$, to stimulate the synthesis and release of pro-inflammatory cytokines. The MAPK signalling, controls a wide range of cellular activities of the innate response and they are particularly important on the regulation of pro-inflammatory cytokine gene expression and programmed cell death [3]. The best characterized MAPK families are: (i) ERK 1 and 2, the extracellular regulated signal protein kinases; (ii) JNK 1, 2 and 3, the c-Jun amino terminal kinases and (iii) MAPK p38, in charge of the onset of innate immune response [4]. Other important group of PRRs is the Nod-like receptor (NLR) family, which is present primarily in macrophages and dendritic cells and is composed of three domains: the C-terminal region in leucine-rich repeats (LRR), the central nucleotide domain NACHT (NOD) and the N-terminal domain that have either a caspase recruitment domain (CARD) or a pyridine domain (PYD). NLRs task is responsible for the constitution of outstanding protein complexes with enzymatic activity denominated inflammasomes, in charge of the launching of the inflammatory process by further secretion of IL-1 $\beta$ and IL-18. The mechanism used by pore forming toxins to induce the innate response, involves further assembly of the inflammasome and it is a very exciting subject, still under study.

Pore-forming toxins (PFT) have exciting membrane damage processes, they usually consist of soluble monomeric proteins that later assamble to produce the holotoxin, inducing perforation of the cytoplasmic membrane behaving as a chloride channel. Due to its very successful pathogenicity mechanism they are produced by a large variety of pathogenic bacteria. In turn the damaged membrane starts cellular rescue mechanisms, that when unsuccessful, cause profuse diarrhea in patients. The Vibrio cholerae cytolysin (VCC) formerly known as hemolysin, turned out to be a very conserved protein, a very efficiently synthesized PFT, exported by this gramnegative bacterium. The monomeric VCC is released as a $65 \mathrm{kDa}$ water soluble mature protein; depending on the strain, its production may be very efficient, in some cases it is a very actively secreted toxin. Once released to the bacterial surroundings, due to its transmembrane domain the monomeric form of VCC commonly gets its way to insert itself on the cytoplasmic membrane of the target cell. In the cytoplasmic membrane, monomers follow one to another, until forming a molecular pore of approximately 1-2 $\mathrm{nm}$ in diameter [5-7], a self-assembled heptameric holotoxin, in the shape of a $\beta$-barrel structure. Numerous heptameric pores, get to disrupt the membrane permeability $[8,9]$. Each heptameric pore means an injury to the cell, when injuries are multiple, eventually they trigger endocytosis, followed by autophagy as a cell surviving mechanism, leading to the striking vacuolating effect we all know. This process is eventually heading to apoptotic cell death $[10,11]$. The innate immune response of the toxin, has been studied usually in murine models of infection with and without the toxin, using knock out mutants. In efforts to address in vitro the innate response to this cytotoxin, the oligomeric form of the VCC, was studied by self-assemble of heptameric pores on liposome membranes, then used to stimulate murine monocytes and macrophages in vitro showing pro-inflammatory response [12]. Although it was a good start, testing the monomeric form of the VCC in human macrophages treated in vitro, at least in our opinion, would be the most direct way to observe an innate response, in the way it would occur at very early stages of infection in patients.

Still, VCC is an exciting subject that entertains more possibilities involved in the process of Vibrio cholerae bacterial pathogenesis, not only by causing vacuolization or cell lysis, but also by starting a pro-inflammatory innate immune response in macrophages. 
In the present study, the monomeric form of VCC released by the bacteria, was used in dose response experiments until reaching a working concentration that allowed us, avoiding vacuolization, still observe early changes on the MAPK activities attributed to treatments with the cytotoxin. Those changes were determined by performing kinetics of protein activation using Western blots with specific antibodies recognizing MAPks p38 and ERK. In order to support the observed increments of the MAPK proteins, transcriptional activation of the regulating factors responsible for the innate immune response and survival: NF- $\mathrm{BB}$ and AP-1, were explored by real time PCR, resulting in activation of both transcriptional regulators.

\section{Results}

\subsection{Standardization of non-vacuolating conditions for VCC to induce signalling.}

In order to know whether the monomer of VCC was capable of activating a signalling response, treatments of macrophages with non-vacuolating concentrations of the cytotoxin $(42 \mathrm{pg} / \mathrm{ml})$ were studied, determining pro-IL-1 $\beta$ and caspase-1 by western blot (data not show). Pro-IL-1 $\beta$ was present at incubations of 10,15, 30 and $60 \mathrm{~min}$, and not in the control without treatment indicating that the monomeric VCC behaves as a PAMP, stimulating the production of pro-IL-1 $\beta$. Apparently, caspase-1 did not have a face-to-face role at these incubation periods of treatment, under the non-vacuolating concentration (results observed in western blots, data not show).
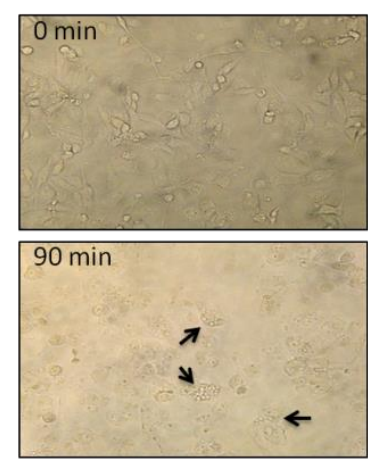

A)

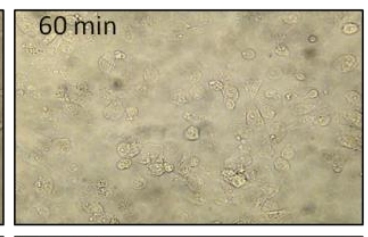

$180 \mathrm{~min}$

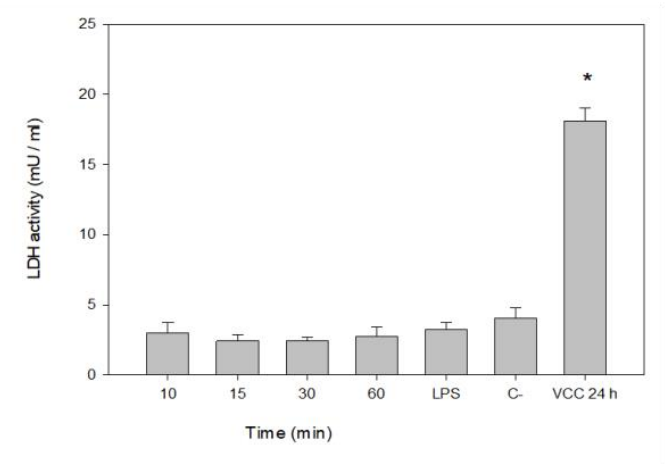

B)

Figure 1. Standardization of non vacuolating but responsive concentrations of VCC. A restraining concentration of VCC (42 pg/ml) is able of inducing release of LDH but not vacuolization under $1 \mathrm{~h}$ incubation periods in THP-1 differentiated macrophages. A) Inverted optical microscopy images where majority of cells are non vacuolated. THP-1 differentiated macrophages were treated with restraining concentration of VCC, in the order of pictograms; they were then monitored and photographed in an inverted optical microscope (40X Olympus IX71 microscope) (0, 60, 90 and 180 $\mathrm{min}$ ). Black arrows indicate characteristic vacuoles (vacuolization) not considered vacuolating effect, at $90 \mathrm{~min}$ incubation with $42 \mathrm{pg} / \mathrm{ml}$ toxin. The results shown are from one experiment and are representative from independent triplicates. B) Plotted results of released LDH from THP-1 cells exposed to VCC. the LDH activity, indirectly indicating necrotic cell death consistent with pyroptosis, was detected and determined from the collected supernatants (times: 0, 10, 15, 30, 60 min and $24 \mathrm{~h}$ ). Data are the mean $\pm \mathrm{SD}$ from four independent experiments. ${ }^{*} \mathrm{P}<0.050$ (ANOVA plus the Student-Newman-Keuls method).

\subsection{VCC is able to activate $p 38$ MAPK.}

A MAPK of great interest is p38, specifically participating on activation of the pro-inflammatory innate immune response, cell differentiation and autophagy of macrophages [13]; consistent with 
our study p38 is activated mainly by PAMPs, pro-inflammatory cytokines and endogenous stress inducers.

Results here support that treatments with VCC during 15 and 30 min induced phosphorylation of p38, with bigger density (1.0 and 0.8 respectively) in comparison to the rest of the evaluated time periods, besides the non-phosphorylated form of p38 is constantly present as a baseline in the treated macrophages (Figure 2A). When the relative density of the bands was plotted (Figure 2B), a Gaussian curve peaking at 15 min was evident. In these experiments, is evident that the untreated control of THP-1, does not show any p38 phosphorylated baseline signal, as it actually happens in the case of phospho-ERK; this fact indicates that no baseline of phosphorylated p38 in macrophages, and therefore all activation observed is directly related to the treatment with VCC.

In the literature, activation of p38 has been reported related to loss of intracellular postassium attributed to cell membrane disruption [14-16], better consistent with the pore forming activity, previously repoted by VCC at concentrations in the order of $\mathrm{ng} / \mathrm{ml}$. This study was designed to avoid the pore formation, which takes place at a thousand times higher toxin concentration, therefore theoretically under this conditions $(42 \mathrm{ng} / \mathrm{ml})$, the stability of the cell membrane is well kept, so activation of $\mathrm{p} 38$, is actually related to a response originated from receptors that trigger the innate immune response.

Consistent with our findings, Porta in 2002 studying pore froming toxins, concludes that activation of p38 will depend on the type of pore forming toxin and on the target cell, that may result in triggering of different mechanisms involved in protection against PFT, and in few cases in activation of programmed cell death responses [15].

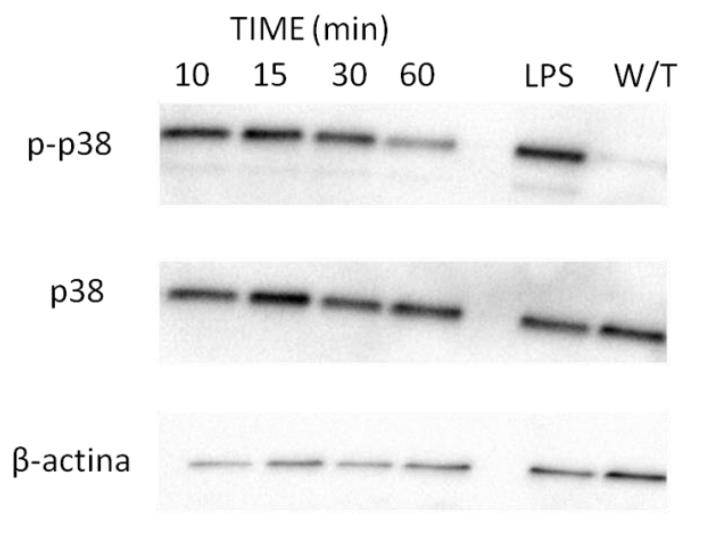

A)

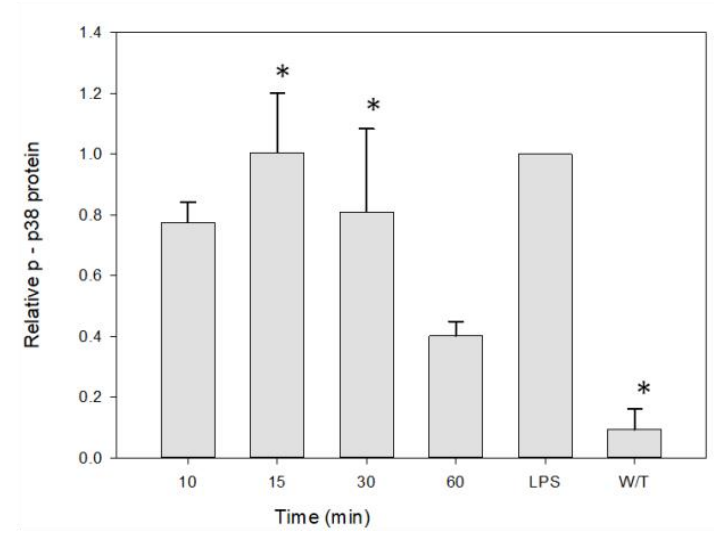

B)

Figure 2. VCC activates the phosphorylation of p38 in THP-1 macrophages. THP-1 macrophages were treated with $42 \mathrm{pg} / \mathrm{ml}$ of VCC at 10, 15, 30 and $60 \mathrm{~min}$. A positive control of LPS (1 $\mu \mathrm{g} / \mathrm{ml})$ was used. Cell lysates were analyzed by the Western blot assay. A) Phosphorylation of p38 after treatments with VCC. The results illustrated are a single experiment representing three separate experiments. In the upper pannel of the Western blot, changes in phosphorylation of P-p38 were identified, while in the middle panel it is observed that the content of non phosphorilated p38 apperars constantly in all the analyzed samples. The lower panel shows the control of homogeneous amount of protein loaded. B) Results in the graphic are representative of 4 performed experiments. $\mathrm{W} / \mathrm{T}$, without treatment. Mean $\pm \mathrm{SD} .{ }^{*} \mathrm{P}<0.050$ (ANOVA plus the Student-Newman-Keuls method).

\subsection{VCC activates the ERK signalling protein.}

Since activity of the expected MAPK signalling starts in a matter of minutes, in this study the periods of interaction of VCC with differentiated THP-1 cells were studied in lapses under $1 \mathrm{~h}$. Reports from colleagues that studied the VCC immune response in mice using mutants, let us know 
that the MAPK signalling pathway is in fact involved in several aspects of the initiation phase of innate immune response [16]. In turn, ERK protein is one of the main activation pathways of MAPKs, it holds two known isoforms: ERK 1/2 also referred as p44 / p42.

Results from treatments with VCC, show that 15 and 30 min after addition of the toxin, ERK1/2 phosphorylation is higher (relative density of 0.6 and 0.2 , respectively) compared to the incubation periods of 10 and $60 \mathrm{~min}$ (relative density of 1.8 and 1.2 respectively); although ERK 1/2 phosphorylated protein is present at all times as a baseline, even without treatment (Figure 3A). The relative density graphic (Figure 3B), shows a Gaussian distribution where the highest point of ERK phosphorylation, occur at $15 \mathrm{~min}$ incubation with VCC. Besides the negative control also show phosphorylated ERK, and it may indicate that there is a preexisting baseline of phosphorylated protein concentration, that prehaps may not be significant.

ERK phosphorylation is directly involved in the cascade of activation of the transcriptional response of pro-inflammatory proteins such as IL-1 $\beta$ and IL-18. Besides regulation of the immune response, ERK is also involved in cell growth and tissue remodelling [17]. It seems that the main role of ERK is regulation of cell proliferation, differentiation and cell death by apoptosis $[18,19]$.

Results of Figure 3 suggest that treatments with the monomeric VCC, would be probably involved with initiating immunomodulatory activity in THP-1 macrophages, they perhaps also may be the start of a later activation of the inflammasome, involving programmed cell death with participation of Caspase-1, characteristic of pyroptosis.

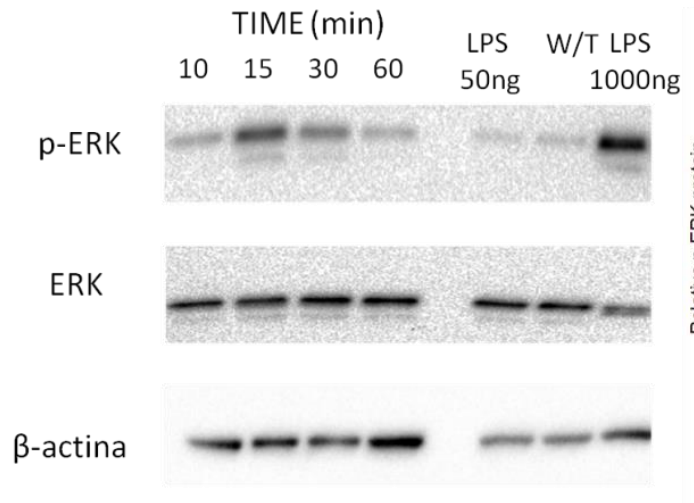

A)

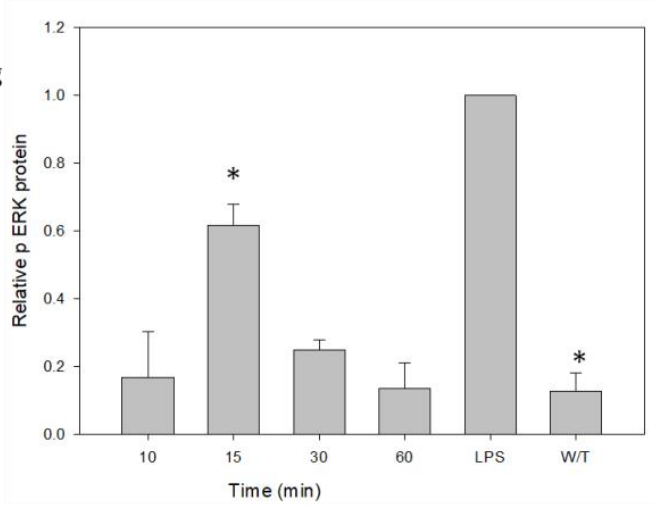

B)

Figure 3. VCC activates the phosphorylation of ERK in THP-1. THP-1 cells were treated with the VCC at 10, 15, 30 and $60 \mathrm{~min}$. As a positive control LPS $(1 \mu \mathrm{g} / \mathrm{ml})$ was used. Cell lysates were analyzed by Western blot assays. A) Western blot showing ERK phosphorylation under treatments with $42 \mathrm{pg} / \mathrm{ml}$ of VCC at 10, 15, 30 and $60 \mathrm{~min}$. The results illustrated are a single experiment representative from three; changes in the phosphorylation of p-ERK are identified in the heading of the Western blot, below it is shown that the ERK content is constant in all the samples analyzed. Actin is the experimental control of protein content. B) Kinetic of treatment with VCC. The graphic includes the results from 4 performed experiments. W/T, without treatment. Mean \pm SD. ${ }^{*} \mathrm{P}<0.050$ (ANOVA plus Student-Newman-Keuls method).

\subsection{VCC monomer triggers activation of AP-1 in THP-1 macrophages.}

Activator Protein-1 (AP-1) transcription factor, is linked to the MAPK activation pathway, it's a heterodimeric protein formed by Fos and Jun. To find out whether the VCC monomer was able to activate the AP-1 transcription factor, mRNA expression of the dimers Fos and Jun, were determined in the VCC treated THP-1 macrophages, at the same time periods previously studied. 
RT-PCR results showed that the monomeric-VCC induces a raise in the transcription of fos and jun genes, at 30 and $60 \mathrm{~min}$ of treatments, compared 10 and $15 \mathrm{~min}$ or the control without treatment (Figure 4), suggesting that AP-1 is actively functioning.

The transcription factor AP-1 regulates a range of cellular processes such as cell proliferation, differentiation and apoptosis [20]. In turn, JNK is a MAPK present in multiple cell types and has an important role in the innate immune response. JNK is known to activate inflammatory cytokines such as IL-1 $\beta$ [21].

Professor B. Khilwani [22] in 2015 studied the immune response towards the heptameric VCC; he used artificially assembled heptamers, the oligomeric VCC, assembled into artificial membranes (liposomes). Under those conditions RAW264.7 and THP-1 cells, he observed induction of pro-inflammatory responses dependent of JNK-mediated activation of AP-1, characterized by the nuclear translocation of subunits c-fos and c-jun. Experiments from Professor Khilwani, are in concordance with observations here of an increase in the transcription of Jun and Fos, at 30 and 60 $\mathrm{min}$, that may be interpreted as macrophage starting activation, not only of survival signals but also pro-inflammatory ones [22].

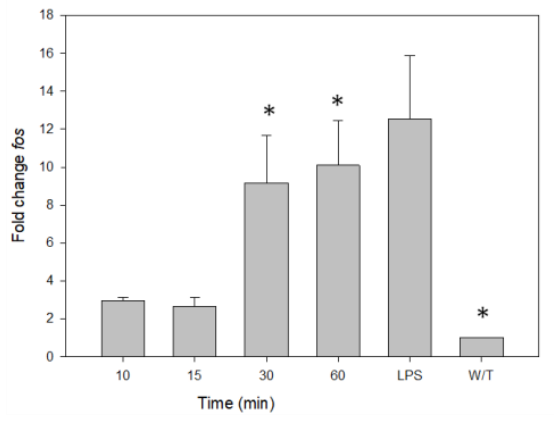

A)

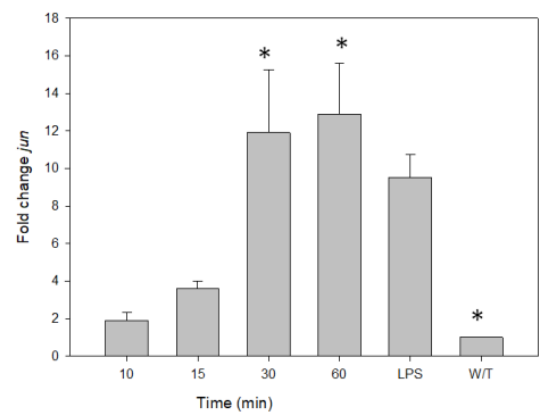

B)

Figure 4. The VCC initiates messenger RNA expression of the transcription factor AP-1. Kinetics of transcriptional activation in THP- 1 cells were performed by treatments with $42 \mathrm{ng} / \mathrm{ml}$ VCC during 10, 15, 30 and $60 \mathrm{~min}$. LPS $(1 \mu \mathrm{g} / \mathrm{ml})$ was the positive control. A) Transcriptional activation of Fos. VCC starts transcriptional regulation of sub units of AP-1 Fos and Jun, then its mRNA expression was studied by qp-PCR. B) Transcriptional activation of Jun. VCC starts transcriptional regulation of sub units of Jun and its mRNA expression was evaluated using qp-PCR. Changes in transcriptional expression were compared with the untreated control, then normalized with the housekeeping gene NAPDH. Results shown here are representative of three different experiments. $\mathrm{W} / \mathrm{T}$, without treatment. Mean $\pm \mathrm{SD} .{ }^{*} \mathrm{P}<0.050$ (ANOVA plus Student-Newman-Keuls method).

\subsection{VCC monomer also triggers activation of NF- $\kappa B$ in THP-1 macrophages.}

As it is well known, the transcription factor NF- $\kappa B$ has an essential role in the expression of pro-inflammatory mediators, it is composed of two subunit heterodimers, p65/p50. As we examine the possibility of support that the VCC monomer is capable of activating the NF- $\kappa \mathrm{B}$ transcription factor, we measure the amount of p50 protein-mRNA expressed in THP-1 macrophages treated with the toxin.

Quantitation of expression of NF-kB (p50) by RT-PCR indicae that THP-1 cells, treated with 42 $\mathrm{pg} / \mathrm{ml}$ of VCC (Figure 5) show a maximun increase of p50 expression at $60 \mathrm{~min}$. It has been noticed that the positive control stimulated with LPS, p50 expression is even greater than the VCC stimulated experimental treatment, after the longest incubation of $60 \mathrm{~min}$. 
Consistent with these results, signaling pathways extrinsically started through p38, ERK and the transcription factor NF- $\kappa \mathrm{B}$, by PAMPs such as LPS, have been associated with further assembly of the inflammasome and enhanced synthesis of pro-IL-1 $\beta$ [23]. The increase in the transcription of NF-kB-p50 after 60 min observed in these experiments, may be interpreted as a VCC elicited response of the macrophage, starting the innate pro-inflammatory cascade associated to the activation of the multiprotein complex machinery of the inflammasome, perhaps already self-ensembled.

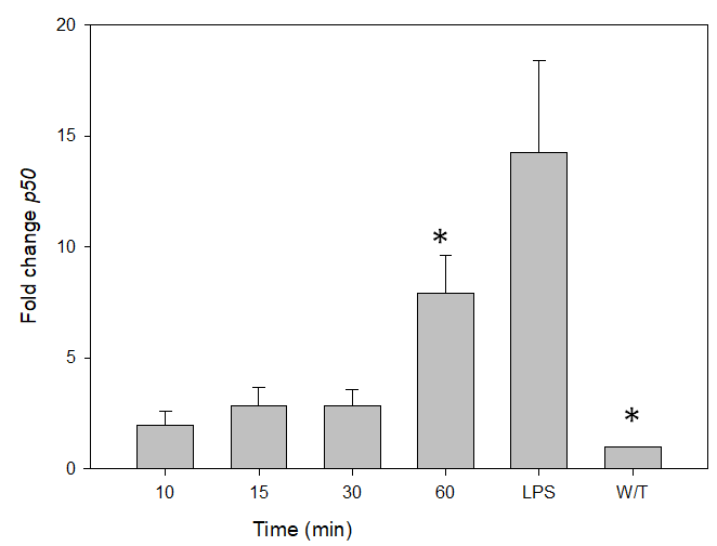

Figure 5. The VCC initiates the expression of the transcription factor NF- $\mathrm{B}$. THP-1 cells were treated with the VCC toxin at the times of 10, 15, 30 and $60 \mathrm{~min}$. LPS $(1 \mu \mathrm{g} / \mathrm{ml})$ was used as a positive control. The VCC starts transcriptional regulation of sub units of NF- $\kappa B$, p50. The mRNA expressions were evaluated using qp-PCR. Changes in expression were compared with the control without treatment and normalized with the housekeeping gene NAPDH. The results shown are representative of 3 different performed experiments. W/T, without treatment. Mean $\pm \mathrm{SD}$. ${ }^{*} \mathrm{P}<0.050$ (ANOVA plus Student-Newman-Keuls method).

\section{Discussion}

TLRs were expected to activate the transcription factors NF- $\mathrm{KB}$ and AP-1, through MAPKs signaling cascade pathways, so this study takes for granted that the VCC stimulates one or more TLRs. Here it is proposed a model in which the VCC monomer is detected by a signaling receptor TLR (based on the literature TLR4 or TLR2/TLR6) [22, 24], which in turn trigger the signaling cascade activating p38 MAPKs and ERK, further triggering transcriptional activation of fos / jun proteins, conforming the transcription factor AP-1, and it also activates the transcription of p50, which is part of the heterodimeric transcription factor NF- $\kappa$ B. Both transcription factors are implicated in the inflammatory response, therefore the monomeric VCC alone is capable to start an inflammatory response depending on intraction with membrane receptors responding to PAMPs.

Results reported by Tetsuya [25] are consistent with this study. KO-p38 baby hamster kidney cells were assayed with Aerolysin, the PFT produced by Aeromonas and the cell death rate increased at least 4-5 times higher, in comparison to the parental cells [26]. These authors found that part of the transcriptional response towards PFTs is functionally important for defense, it is specifically responsive to PFTs and not the whole bacteria. To our delight, results from this study concord with those found using the whole bacteria [26], two of the transcriptionally induced MAPKs were p38 and JNK; alas, the reason that both p38 and JNK are required to protect the cell in front of PFTs is still under debate, so a proposal will be offered.

It is our belief that results from this work, support that the VCC monomeric protein, at very low concentrations, like in the onset of an infection, is initially alerting the macrophage, very probably stimulating the signaling from TLRs or NLRs (Figure 6). The treatments with VCC shortly starting responses, make obvious that at least in macrophages, the most likely response to be stimulated is in 
charge of $\mathrm{p} 38$, since production of innate immunity cytokines like IL-1 $\beta$ would be the main goal of the first line of innate immune protection cells. As important as it might be the innate immune response in charge of p38, the toxin also initiates cell death by the pyroptotic pathway (with activated Caspase-1, result not shown) this feature can be indirectly recognized by the leaking of $\mathrm{LDH}$ determined after $24 \mathrm{~h}$ of the treatment, in the conditioned media (Figure 7 ). From the above reasoning, "protection" of the cell towards PFTs, as it was suggested by our colleagues, at least in this case, is not as worthy for a hypothesis. Instead, results here show that with monomeric VCC, ERK (and perhaps JNK), are activated almost at the same time with p38, (however, within the first hour of incubation). Since, JNK is known to lead a cell survival response [26], actually, activation of ERK turns out to be a little paradoxical, since it is not a kinase related to pyroptosis or neither to enhancement of the innate immune response, or as it is in our hands, activated by treatments with monomeric PFTs such as VCC. Activation of ERK, is actually a survival response that only makes sense, once it is associated to a rather prolonged survival of a pyroptotic cell, very possibly to ensure optimal release of pro-inflammatory cytokines such as IL-1 $\beta$ actively produced by the inflammasome. In other words, the innate immune response, remains in charge of the p38 pathway; so that the innate immune cytokines are profusely synthesized by the inflammasome, and this response is kept going, as long as possible, sustained due to the survival response leaded by ERK and JNK. ERK and perhaps JNK pathways, in the particular case of activated macrophages responding towards a pathogenic cytotoxin, rely on activation of the survival signals before necrotic cell death strike, (Figure 7) meanwhile, cytokines of the innate response, effectively convey the message to cells of the second line of protection, such as leukocytes but importantly, to the $\mathrm{T}$ lymphocytes in charge of generating the specific immune response.

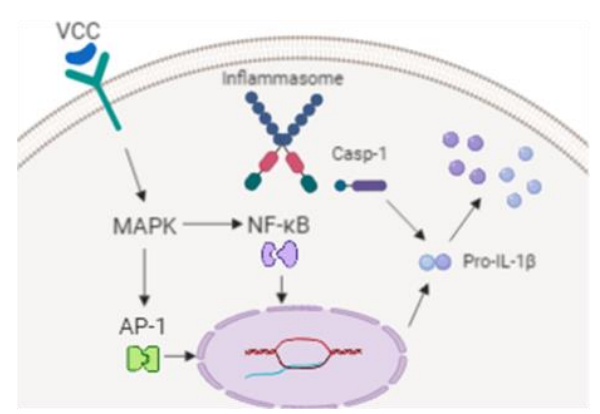

Figure 6. Model mechanism proposed for the monomeric VCC, starting an innate immune response. The monomeric form of the VCC behaves as a PAMP, it binds its membrane receptor (perhaps TLR 4), turning on a signaling cascade activating MAPKs (ERK, p38 and JNK). The MAPKs then initiate activation of transcription factors AP-1 and NF- $\kappa \mathrm{B}$, begins the production of pro-inflammatory innate response involving increase of the synthesis of the classic IL- $1 \beta$. 


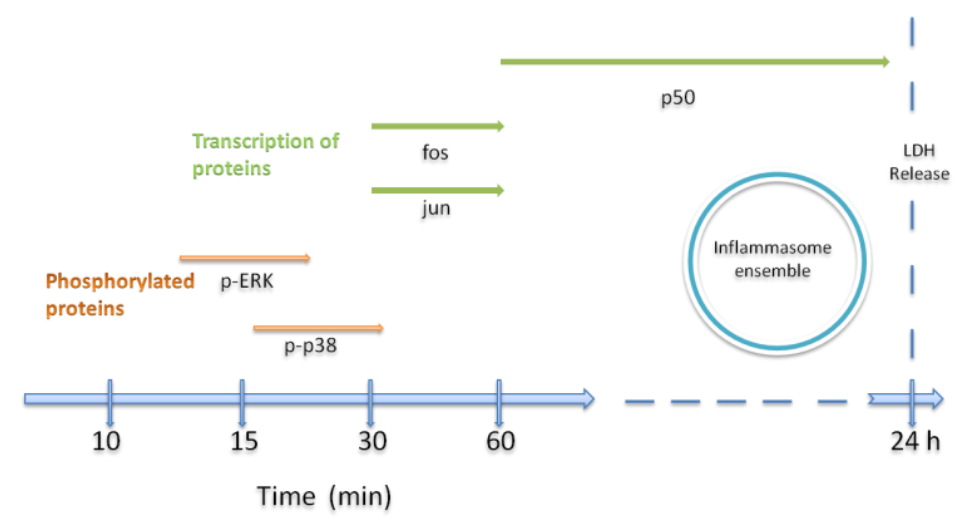

Figure 7. Propposed time lapsed of activated MAPKs and their transcriptional activators. Phosphorylated MAPK-proteins and activated transcripts of Fos, Jun and NFkB elicited by treatments with the monomeric VCC on THP-1 treated cells.

\section{Conclusions}

This is the earliest study standing the basis of the use of small concentrations (pg/ml) of the VCC, to ensure it is kept in so limited availability, that achieved activation of the MAPKs and transcription factors, will take place in the absence of cytoplasmic vacuolization, and only may obey to interaction with membrane receptors such as TLRs, under the conditions here used.

Experimental results obtained in this study strongly suggest that the monomer of $V$. cholerae cytotoxin VCC, is capable of activating the main pathways of MAPK, initiating a pro-inflammatory response and immediately followed by a surviving response. Here it is also shown that non-vacuolating concentrations of VCC, in the order of picograms of the toxin ( $42 \mathrm{pg} / \mathrm{ml}$ was used), were applyed to THP-1 macrophage differentiated cells, and they exhibited a survival response evidenced by ERK activation, and therefore activation of the AP-1 transcription factor is also observed. Subsequently, the macrophage initiate the pro-inflammatory response, evidenced by p38 activation, triggered by NF- $\mathrm{BB}$ transcription factor. Perhaps longer exposure treatments are needed to identify assemble and activation of the inflammasome, those experiments are already being assayed. Authors in $[22,24]$ previously reported that VCC, is sensed by TLR4 or TLR2/TLR6, so although this work did not dissect or point out the particular TLR starting the MAPK signaling activation and later transcriptional activation of NF- $\mathrm{KB}$ and $\mathrm{AP}-1$ in human macrophages, experiments determining the above interesting questions are already in process. Finaly, in any case, further studies are needed to identify whether the recognition receptor may be perhaps TLR4 [27], since the signalling is started at times shorter than $1 \mathrm{~h}$ (as described for LPS responses).

\section{Materials and Methods}

\subsection{Chemical agents and antibodies}

Antibodies against the kinase activated by extracellular receptor (ERK) 1/2, phosphorylated ERK, p38, phosphorylated p38, caspase-1 and interleukin-1 were all obtained from Cell Signalling and $\beta$-actin was obtained from Santa Cruz Biotechnology. The kit of reagents for determination of releasing of $\mathrm{LDH}$ was obtained from Biovision.

\subsection{Preparation of the VCC toxin}

The strain of Vibrio cholerae 69750 which is a super producer of VCC previously reported in [10]. Bacteria was grown in $5 \mathrm{ml}$ of LB medium at $37^{\circ} \mathrm{C}$ with a constant agitation of $270 \mathrm{rpm}$ overnight. 1 $\mathrm{ml}$ of supernatant was taken from the cells and centrifuged at 14,000 x $\mathrm{g}$ for $1 \mathrm{~min}$ in a micro-centrifuge at $4^{\circ} \mathrm{C}$. Subsequently, the supernatant was collected and sterilized by a $0.22 \mu \mathrm{m}$ 
membrane filter, finally the toxin was concentrated by molecular weight exclusion columns (100 $\mathrm{KDa}$ and then $50 \mathrm{kDa}$ of Amicon Ultra 0.5 Ultrafiltration system at 14,000g for $10 \mathrm{~min}$ ), the concentrated toxin was identified by $12 \%$ PAGE, then collected and preserved at $-4^{\circ} \mathrm{C}$ until its use. Biologic effect of the final toxin preparation was evaluated by dose response experiments.

\subsection{Cell culture}

The human monocyte cell line THP-1 was obtained from ATCC and donated by the Department of Biochemistry of the National Institute of Nutrition and Medical Sciences, generously provided by Dr. Sigifredo Pedraza Sánchez. The cells were cultured as recommended, in RPMI 1640 medium supplemented with $2 \mathrm{mM}$ L-glutamine, $10 \mathrm{nM}$ HEPES, $1 \mathrm{mM}$ sodium pyruvate, $0.05 \mathrm{mM}$ $\beta$-mercaptoethanol and $10 \%$ fetal bovine serum. The cells were incubated at $37^{\circ} \mathrm{C}$ in a humidified atmosphere with $5 \% \mathrm{CO}^{2}$. For the tests, 6 -well plates were used in which $1.5 \times 10^{6}$ cells per well were used and $50 \mathrm{ng} / \mathrm{ml}$ of PMA were added, in $2 \mathrm{ml}$ of RPMI 1640 supplemented medium. Subsequently the cells were incubated at $37^{\circ} \mathrm{C}$ in a humidified atmosphere with $5 \% \mathrm{CO}^{2}$ for 48 hours, they were exchanged for fresh medium and re-incubated at $37^{\circ} \mathrm{C}$ in a humidified atmosphere with $5 \%$ overnight $\mathrm{CO}^{2}$, old medium was removed. New medium was added, this time using supplemented RPMI, without phenol red and without FBS.

\subsection{Titration of Biologic activity (Vacuolating effect)}

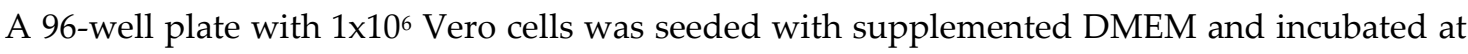
$37^{\circ} \mathrm{C}$ in humidified atmosphere with $5 \%$ overnight $\mathrm{CO}^{2}$, then fresh medium was added to the cells without FBS. One volume of the concentrated toxin was added to the first well of the plate, then 1:2 serial dilutions were made in the following wells. Then the plate was incubated at $37^{\circ} \mathrm{C}$ in a humidified atmosphere with $5 \% \mathrm{CO}^{2}$ and was observed by inverted optic microscopy every $30 \mathrm{~min}$ and beware for the vacuolating effect on the cells, as reported in Figueroa-Arredondo et al 2001 [10].

Determination of protein concentration of the VCC was performed by the Bradford Method as described in [28]. The concentration of VCC to be used is determined by its biologic activity, the shorter concentration showing at least $50 \%$ of the cells with heavily vacuolated cytoplasm is considered a vacuolating concentration as established in Figueroa-Arredondo et al 2001 [10]. In this work, the sub-vacuolating cytotoxin concentration is determined by the lowest concentration do not producing vacuolating effect $24 \mathrm{~h}$ after addition of the toxin, but instead LDH is released to the cell culture medium.

\subsection{Kinetics of the VCC in THP-1 cells}

THP-cells already differentiated were treated with the sub-vacuolating concentration of VCC standardized as referred above, incubating the following periods 10, 15, 30 and 60 min respectively. The positive control was $1 \mathrm{mg}$ of LPS; the negative control were cells with no treatment. After incubation, the supernatants were collected and stored at $-80^{\circ} \mathrm{C}$ and the cells were suspended in 200 $\mu \mathrm{l}$ of RIPA lysis buffer and the plate was scraped with cell lifters, the supernatant was collected and centrifuged at $14,000 \mathrm{xg}$ for $10 \mathrm{~min}$ at $4^{\circ} \mathrm{C}$. The protein concentration was determined by the method (Pierce BCA protein assay kit).

\subsection{Immunoblot}

Collected cells from the treatments above were kept frozen at $-80^{\circ} \mathrm{C}$ in lysis buffer added of a cocktail of protein (cOmplete Lysis), from here $10 \mu \mathrm{g}$ of protein from each treatment was loaded in a pre-made denaturing electrophoresis gel of $10 \%$ acrylamide (invitrogen Novex) and electrophoresed $15 \mathrm{~min}$ at $80 \mathrm{~V}$ and then electrophoresed forward $75 \mathrm{~min}$ at $150 \mathrm{~V}$. Electrophoresis separated protein bands were transferred to a polyvinylidene fluoride membrane (Invitrogen Novex 
PVDF membrane), then pre-treated with $5 \%$ bovine serum albumin in Tris buffer plus $0.1 \%$ Tween 20 at room temperature by $1 \mathrm{~h}$. Blotted membranes were incubated overnight in the primary antibody at $4^{\circ} \mathrm{C}$, then subsequently incubated with the secondary antibody by $1 \mathrm{~h}$ at room temperature. Luminol was used in the detection of electrophoretic bands and analyzed on a photo-documenter (ChemiDoc XRS+). Relative densities from the bands were estimated with the Imagelab software. Antibodies used in this study were mentioned above.

\section{7. $L D H$ test}

Supernatants from each kinetic assay $(25 \mu \mathrm{l})$ were taken and added to a 96-well plate and $25 \mu \mathrm{l}$ of the working solution was added to each well. The appropriate standard curve of LDH and the working solution were made according to the manufacturer's instructions (Biovision). The optical density was measured at $450 \mathrm{~nm}$ (time zero) followed by incubation at $37^{\circ} \mathrm{C}, 30 \mathrm{~min}$ with constant shaking $(300 \mathrm{rpm})$. Optical density $(450 \mathrm{~nm})$ was measured after $30 \mathrm{~min}$ period of activity. LDH activity of from every treatment was determined according to the formula provided by the manufacturer. These measurements were performed in duplicates from three different experiments and graphics constructed from the collected data.

\section{8. $q R T-P C R$}

The sub-vacuolating concentration of VCC was added to $1 \times 10^{6}$ THP-differentiated cells, followed by incubation by 10, 15, 30 and $60 \mathrm{~min}$ respectively. The positive control was $1 \mathrm{mg}$ of LPS, the negative control were the cells without treatment. Total RNA was extracted using Roche's High Pure RNA Tissue kit, then cDNA from each treatment was synthesized from approximately $500 \mathrm{ng}$ of RNA using Roche's Fisrt Strand cDNA Synthesis Transcription Kit. The generated cDNA was used to perform duplicates of qRT-PCR assays using Roche's Light Cycler TaqMan Master. The primers used were the following: p50_Fwd:5'-caccgaagcaattgaagtga-3', p50_Rev:5'-ggcctgagaggtggtcttc-3', jun_Rev:5' -ctgtccetctccactgcaac-3', jun_Fwd:5' -ccaaaggatagtgcgatgttt-3', fos_Fwd:5' -ctaccactcaccegcagact-3', fos_Rev:5'-aggtccgtgcagaagtcct-3', gapdh_Fwd:5'-agccacatcgctcagacac-3', gapdh_Rev:5'-gcccaatacgaccaaatcc-3'. Glyceraldehyde-3-phosphate dehydrogenase (GAPDH) was used as a housekeeping control gene. All reactions were performed in the LightCycler 2.0 (Roche) real-time equipment, and the relative transcription results were normalized against GAPDH using the $\Delta \Delta \mathrm{Ct}$ method [29].

\subsection{VCC Super-producer V. cholerae 69750}

In the beginning of the study it was necessary to purify the VCC to make sure that the toxin is the one molecule interacting with the cell line macrophages, so the $V$. cholerae 69750 strain [10] was cultivated for over expression of the VCC. Two aliquots $(1 \mathrm{ml})$ from the supernatant of the cultures of $V$. cholerae were taken, both aliquots filter sterilized $(0.22 \mu \mathrm{m})$. Since the VCC toxin in its monomeric form has a molecular weight of $65 \mathrm{kDa}$, a $50 \mathrm{kDa}$ ultracentrifugation column was used to keep molecules greater than $50 \mathrm{KDa}$. An electrophoresis in acrylamide-bis was performed, to assure that purification has been carried out successfully. Three conditions were electrophoresed, the $50 \mathrm{kD}$ column concentrate, the filtered supernatant and sterile LB medium. As expected, a band corresponding to $65 \mathrm{kD}$ is observed in the lane where the concentrate is loaded, corresponding to the VCC molecular weight. In the lane where the filtered supernatant is loaded, a variety of different bands were observed, indicating that the VCC was purified (results not shown). For corroboration, an anti-VCC specific antibody (kindly provided by Professor Takeshi Honda) was used, the observed $65 \mathrm{KDa}$ protein corresponding to the VCC in western blot, was identified ruling out the possibility of having a mistaken some other protein with similar molecular weight.

\subsection{Standardization of signalling responsive concentration of VCC}


In order to define the optimum concentration of the VCC to be used in signalling experiments, that specifically avoid the vacuolating effect more related to autophagy [30], standardization of a sub-vacuolating concentration was empirically determined, initially the use of the Vero cell line was considered to be convenient, since the vacuolating effect was previously characterized in this cell line [10]. The sub-vacuolating concentration was estimated to be the one where no vacuolating effect was observed after $1 \mathrm{~h}$ and neither after $12 \mathrm{~h}$ of incubation. Serial dilutions were performed until such concentration was experimentally reached.

From the VCC concentrated stock, different concentrations were tested, as described above. By optical microscopy after $1 \mathrm{~h}$ was observed that at the concentrations of the order of $\mathrm{ng}(42 \mathrm{ng} / \mathrm{ml})$ show the typical vacuolating effect (Figure 1A), however at concentrations of the order of pg (42 $\mathrm{pg} / \mathrm{ml}$ ) not any vacuolating effect is observed after $12 \mathrm{~h}$. Instead, LDH was detected from the treated cell supernatants after $24 \mathrm{~h}$, therefore the concentration of $42 \mathrm{pg} / \mathrm{ml}$ was selected, since the goal of this project is to study sub-vacuolating toxin concentrations only capable to start signalling cascades towards activation of the MAPK pathways.

Previous studies reported the cytotoxin of $V$. cholerae being able to induce innate immune response through ensemble and activation of the inflamasome protein complex [24, 31] leading to pro-inflammatory cell death denominated pyroptosis [32]. To determine if the monomer VCC was able to induce such type of cell death, presence of LDH released in the treatment supernatants was determined at 10, 15, 30 and 60 min of treatments with the VCC and after $24 \mathrm{~h}$ treatment (Figure 1B). LDH unequivocally indicates lysis of the treated cells, therefore pyroptosis is highly probable to occur.

Funding: Julio Rodrigo Escartín Gutiérrez is a Fellow of Beca de Estudios de Doctorado financed by the National Council of Science and Technology, Conacyt. Mexico and was awarded the BEIFI-Instituto Politécnico Nacional scholarship.

Drs. Paula Figueroa and Aldo Resendiz wish to acknowledge partial financing support to this work, from Secretaría de Investigación y Posgrado, Proyectos SIP 2016 1341, 2017 2268, 2018 1796, 20194973.

Acknowledgments: This work is dedicated to our beloved friend Professor Dr. Rafael Campos Rodríguez, the Academic and moral leader of people working in his Lab and even more. The world will never be the same without him, but he would have been proud to see this paper published.

We wish to acknowledge the kindness of Laboratorios de Especialidades Farmacéuticas (LEI) and specially our former co-worker Dr. Laura Padierna Mota since envery time we reached for help in availability of resources, she was ready to help solve the problems. Thank you Laurita, we love you.

Conflicts of Interest: The authors declare no conflict of interest.

\section{References}

1. Beutler, B. Innate immunity: an overview. Molecular Immunology. 2004, 40, 845-859.

2. Akira I S.; Uematsu, S. Takeuchi, O. Pathogen Recognition and Innate Immunity. Cell. 2006, 124, 783-801.

3. Takeda, K.; Akira, S. Toll like receptors in innate immunity. International Immunology. 2005, 17, 1, 1-14.

4. Cargnello, M.; Roux P. P. Activation and Fuction of the MAPKs and their substrates, the MAPK-Activated Protein Kinases. Micro. and Mol. Biol. Rev. 2011 , 75, 1, 50-83.

5. Paul, K.; Chattopadhyay, K. Pre-pore oligomer formation by Vibrio cholerae cytolysin: Insights from a truncated variant lacking the pore-forming pre-stem loop. Bioch and Bioph Res Commu. 2013, 443, 189-193.

6. Kathuria, R.; Chattopadhyay, K. Vibrio cholerae cytolysin: Multiple facets of the membrane interaction mechanism of a $\beta$-barrel pore-forming toxin. IUBMB Life. 2018, 70, 4, 260-266.

7. Gutierrez, M.; Saka, H.; Chinen, I.; et al. Protective role of autophagy against Vibrio cholerae cytolysin, a pore-forming toxin from $V$. cholerae. PNAS. 2006, 104, 86, 1829-1834. 
8. Dal Peraro, M.; Gisou van der Goot, F. Pore-forming toxins: ancient, but never really out of fashion. Nature Rev Micro. 2016, 14, 77-92.

9. Iacovache, I.; Bischofberger, M.; Gisou van der Goot, F. Structure and assembly of pore-forming proteins. Current Opinion in Structural Biology. 2010, 20, 241-246.

10. Figueroa, P.; Heuser, J. E.; Akopyants , N. S. et al. Cell vacuolation caused by Vibrio cholerae hemolysin. Infect Immun. 2001, 69, 3, 1613-24.

11. Munafó, D. B.; Colombo, M. A novel assay to study autophagy: regulation of autophagosome vacuole size by amino acid deprivation. Journal of Cell Science. 2001, 114, 3619-3629.

12. Khilwani, B.; Mukhopadhaya, A.; Chattopadhyay, K. Transmembrane oligomeric form of Vibrio cholerae cytolysin triggers TLR2/TLR6-dependent proinflammatory responses in monocytes and macrophages. Biochemical Journal. 2015, 466, 1, 147-161.

13. He, Y.; She, H.; Zhang, T. et al. p38 MAPK inhibits autophagy and promotes microglial inflammatory responses by phosphorylating ULK1. J. Cell Biol. 2018, 217, 1, 315-328.

14. Kloft, N.; Busch, T.; Neukirch, C.; et al. Pore-forming toxins activate MAPK p38 by causing loss of cellular potassium. Biochemical and Biophysical Research Communications. 2009, 385, 503-506.

15. Porta, H.; Cancino, A.; Soberon, M.; et al. Role of MAPK p38 in the cellular response to pore former toxins. Peptides. 2002, 32, 601-606.

16. Yang, Y.; Kim, S. C.; Yu, T. Functional Roles of p38 Mitogen-Activated protein kinase in macrophage-mediated inflammatory responses. Mediators of Inflammation. 2014, 2014, Article ID 352371.

17. Song, Y.; Ding, N.; Kanazawa, T. Curcubitacin D is a new inflammasome activator in macrophages. International Immunopharmacology. 2013, 17, 1044-1050.

18. Cagnol, S.; Chambard, J. C. ERK and cell death: Mechanisms of ERK-induced celldeath - apoptosis, autophagy and senescence. FEBS Journal. 2010, 277, 2-21.

19. Sun, Y.; Lou, W. Z.; Liu, T. Signaling pathway of MAPK/ERK in cell proliferation, differentiation, migration, senescence and apoptosis. J Recept Signal Transduct Res. 2015, 35, 6, 600-604.

20. Garces de los Fayos Alonso, I.; Liang, H.; Turner, S. D.; et al. The role of activator protein-1 (AP-1)family members in CD 30-positive lymphomas. cancers. 2018, 10, 93, doi:10.3390.

21. Zeke, A.; Misheva, M.; Reményl, A.; et al. JNK signaling: Regulation and fuctions based on complexprotein-protein partnerships. Micro. and Mol. Biol. Rev. 2016, 80, 3, 793-835.

22. Khilwani, B.; Mukhopadhaya, A.; Chattopadhyay, C. Transmembrane oligomeric form of Vibrio cholerae cytolysin triggers TLR2/TLR6-dependent proinflamatory responses in monocytes and macrophages Biochem. J. 2015, 466, 147-161.

23. Töszér J.; Benkö, S. Natural compounds as regulators of NLRP3 inflammasome-mediated IL- $1 \beta$ production. Mediators of Inflamm. 2016, 2016.

24. Toma, C.; Higa, N.; Koizumi, Y.; et al. Pathogenic vibrio activate NLRP3 inflammasome via cytotoxins and TLR/Nucleotide binding oligomerization domain-mediated NF- $\kappa B$ signaling. J. Immunol. 2010, 184, 5287-5297.

25. Saitoh, T.; Akira, S. Regulation of inflammasomes by autophagy. J Allergy Clin Immunol. 2016, 138, 1, 28-36.

26. Huffman, D. L.; Abrami, L.; Sasik R.; et al. Mitogen-activated protein kinase pathways defend against bacterial pore-forming toxins. PNAS. 2004, 101, 30, 10995-11000.

27. Chakraborty, D. C.; Mukherjee, G.; Banerjee; P.; et al. Hemolysin induces Toll-like Receptor (TLR)-independent apoptosis and multiple TLR-associated parallel activation of macrophages. The Journal of biological chemistry. 2011, 286, 40, 34542-34551.

28. Kruger, N. J. The bradford method for protein quantitation. Basic protein and Peptide protocols. 1994, 32, 9-16.

29. Livak, K. J.; Schmittgen, T. D. Analysis of relative gene expression data using real-time quantitative PCR and the $2-\Delta \Delta C_{T}$ method. Methods. 2001, 25, 402-408.

30. Gutierrez, M. G.; Saka, H. A.; Chinen, I.; et al. Protective rol of autophagy against Vibrio cholerae cytolysin, a pore- forming toxin from $V$. cholerae. PNAS. 2007,104, 6, 1829-1834.

31. Queen, J.; Agarwal, S.; Dolores, J. S.; et al. Mechanism of inflammasome activation by Vibrio cholerae secreted toxins vary with strain biotype. Infect Immun. 2015, 83, 2496-2506.

32. Ciraci, C.; Janczy, J. R.; Sutterwala , F. S. et al. Control of innate and adaptative immunity by the inflammasome. Microbes and Infection. 2012, 14, 1263-1270. 